\title{
Entre lo público y lo privado: los espacios y sus valores en \\ Los caballeros de la capa, de Ricardo Palma
}

Por Félix Terrones 
Escritor, crítico literario y traductor, doctor en Literatura por la Universidad Michael de Montaigne, de Bordeaux. Actualmente, traduce la novela "Conquistadors" del francés Eric Vuillard. Profesor contratado de la Université Francois Rabelais de Tours (Francia). 
Caídos y levantados, hartos y hambrientos, eso ha sido la colonia y eso es y ha sido la república.

Ricardo Palma, Los caballeros de la capa

\section{Introducción}

Los caballeros de la capa pertenece a la segunda serie de las Tradiciones (1874) que Ricardo Palma publicara en vida. ¿Qué caracteriza a esta segunda serie con respecto de la primera? Si tenemos en cuenta lo afirmado por la crítica francesa Isabelle Tauzin-Castellanos, la segunda serie de las tradiciones no es una "recopilación de textos dispares sino un volumen cuidadosamente estructurado que se atiene a una cronología de modo que el conjunto tiene una nítida orientación historicista, del todo diferente del predominio de la ficción de la Primera serie"1. En ese sentido, podemos considerar que, cuando se trata de la segunda serie, el arte de Ricardo Palma se encontraba en su apogeo tanto formal como ideológico. Lo cual nos lleva legítimamente a preguntarnos en la manera en que dicho arte toma forma literaria, así como las inquietudes y los posicionamientos que lo alientan.

1 TAUZIN-CASTELLANOS Isabelle, Las Tradiciones Peruanas de Ricardo Palma: claves de una coherencia, Lima: Universidad Ricardo Palma, 1999.p.46. 
Con este objetivo interrogaremos una Tradición en particular, titulada Los caballeros de la capa, la cual no solo forma parte de la segunda serie sino que también es el texto que abre el conjunto, lo que muestra la importancia que dentro de su proyecto estético le entregó Ricardo Palma. No era para menos pues, además de ser uno de los mejores logrados en términos estéticos, plantea un momento culminante de la Historia peruana: el del asesinato del primer virrey Francisco Pizarro, y el posterior ajusticiamiento de sus ejecutores por parte de Cristóbal Vaca de Castro. De esa manera, Ricardo Palma coloca a su lector en el momento exacto en el que se termina con el caudillismo que caracterizó los primeros años de conquista y se empieza la instalación del orden colonial. Momento liminar, en el que se entrecruza un comienzo con un final, dicho evento adquirirá en el texto resonancias singulares que pasan de la anécdota a las lecciones sociales e históricas. Si bien se trata de una Tradición escrita según un esquema bastante básico que puede resumirse en introducción, argumento, desenlace, el núcleo argumentativo, la afrenta al honor y su reparación, le entregan un aliento y una densidad bastante logrados. Me gustaría, por eso, detenerme en los tres grandes espacios representados en Los caballeros de la capa (uno por sección). Se trata de una representación cargada de valores dramáticos en la cual, conforme avanza la lectura, se enfatiza el carácter ineluctable de los acontecimientos, tanto para un bando como para otro, así como se va desplazando a espacios abiertos la resolución de la intriga de honor individual y crisis política. 


\section{El espacio de la intimidad vergonzosa}

Dividida en tres partes ${ }^{2}$, la tradición Los caballeros de la capa puede ser leída en función de cada uno de los tres espacios preponderantes que se suceden en concordancia con el ritmo de la intriga; es decir, primero, el solar en el que viven los desdichados caballeros; después, el palacio de Pizarro y; finalmente, el campo de batalla. De hecho, en Ricardo Palma los espacios no son un simple decorado sino que se encuentran alentados por un significado que no por subyacente deja de estar presente, un significado que complementa y enriquece la evolución de la intriga. En ese sentido, basta detenerse en el comienzo de la Tradición donde, mediante la indicación espacial, ya comienza a anunciar el talante del conflicto:

En la tarde del 5 de junio de 1541 hallábanse reunidos en el solar de Pedro de San Millán doce españoles, agraciados todos por el rey por sus hechos en la conquista del Perú.

La casa que los albergaba se componía de una sala y cinco cuartos, quedando gran espacio de terreno por fabricar. Seis sillones de cuero, un escaño de roble y una mugrienta mesa pegada a la pared, formaban el mueblaje de la casa. Así la casa como el traje de los habitantes de ella pregonaban, a la legua, una de esas pobrezas que se codean con la mendicidad. Y así eran en efecto. ${ }^{3}$

En la primera línea, Ricardo Palma subraya la dimensión histórica, mediante la alusión al día y al año, sin que todavía se especifique por qué dicha fecha es importante. No obstante, el autor limeño circunscribe inmediatamente después el espacio a un lugar íntimo, personal; me refiero al solar de Pedro de

2 Las partes son las siguientes: "Quiénes eran los caballeros de la capa y el juramento que hicieron", "De la atrevida empresa que ejecutaron los caballeros de la capa", "El fin del caudillo y de los doce caballeros".

3 PALMA Ricardo, Tradiciones peruanas (Selección), Madrid: Cátedra, 201 1, p.135. 
San Millán, lo cual crea interés en dicho espacio, además de conferirle un valor, un espesor históricos. ¿Qué interés posee dentro del mosaico histórico palmino el solar de Pedro de San Millán?, es una pregunta que queda flotando sin respuesta a la espera del desarrollo narrativo. Por otro lado, si consideramos el final trágico de los caballeros de la capa podemos deducir que Ricardo Palma utiliza la palabra solar tanto en su acepción espacial como en la familiar. En efecto, "solar" también significa "descendencia", "linaje", lo que no deja de manifestar una macabra ironía cuando recordamos que todos los caballeros fueron ajusticiados y que el descendiente de aquel a quien buscaban vengar, es decir Diego de Almagro, también es asesinado al final del texto. Como es evidente, se trata de un significado que en la primera lectura no aparece pero que contribuye, por anfibología retrospectiva, a cargar los espacios con un valor diegético específico.

Cabe añadir que en la descripción del lugar -del mobiliario hasta las vestimentas- se enfatiza un aspecto en especial. Así, muy en la línea de lo que enunciara Roland Barthes con respecto de las descripciones y la carencia total de accidente en ellas ${ }^{4}$, podemos decir que todo contribuye a configurar el drama de quienes viven en la casa. La sucesión escalonada de substantivos y adjetivos no deja, en ese sentido, lugar a dudas: comienza con "mugrienta", continúa con "pobreza" y termina con "mendicidad", como si con esta palabra se resumiera la escasez material y moral planteada por las anteriores. Aquellos

4 Así lo enuncia el mismo Roland Barthes: «La singularité de la description (ou du "détail inutile") dans le tissu narratif, sa solitude, désigne une question qui a la plus grande importance pour l'analyse structurale des récits. Cette question est la suivante : tout dans le récit, est-il signifiant, et sinon, s'il subsiste dans le syntagme narratif quelque plages insignifiants, quelle est en définitive, si l'on peut dire, la signification de cette insignifiance? ».En : BARTHES Roland, L'effet de réel, Communications. 1968, vol 11, n¹1, p.85. 
peruleros que viajaron desde sus pueblos españoles para hacerse de riquezas parecen, más bien, haberse destinado a una situación precaria que no les permitiría cumplir con el sueño buscado ni satisfacer sus ansias de reconocimiento. Finalmente, subrayemos que la cantidad de sillas y escaños que hay en la casa es bastante inferior al número de individuos que viven y se reúnen el ella, asunto que debe ser tomado como una prueba más de su pobreza material pero también como un anticipo de lo que les caracterizará dentro de la sociedad evocada por Palma.

Me refiero al hecho de que solamente exista una capa para todos, situación que al ser más literaria que histórica debería ser leída antes que nada en su valor textual; es decir, en diálogo con la tradición literaria y en función de sus valores al interior de la narración. En efecto, la anécdota según la cual solamente existía una capa para los doce españoles no posee fundamento histórico, tal y como muchos críticos se han encargado de señalarlo. Antes bien, proviene de la cosecha de Ricardo Palma, quien se valió de ella para darle un componente adicional a su relato que no debe ser dejado de lado. Para empezar, el vincular la identificación de la capa con el honor es una ecuación connotada intertextualmente. Basta recordar al Lazarillo de Tormes donde uno de los amos fue un hidalgo arruinado cuya capa mostraba tanto su anhelo de mostrarse digno como su pobreza $^{5}$. Resulta imposible no concebir el diálogo consciente de Ricardo Palma con la tradición picaresca. Diálogo que no

5 Pensamos también en La vida del Buscón de Francisco de Quevedo y Villegas, en particular el célebre episodio de los caballeros "hebenes, güeros, chanflones, chirles, traspillados y caninos" en el cual un grupo de hidalgos que prestan mucha atención a su indumentaria vive en un espacio donde comparten, al abrigo de las miradas extrañas, comida y ropa. En: QUEVEDO Francisco de, La vida del Buscón, Barcelona: Crítica, 1993, p.144-155. Agradezco a Juan Carlos Garrot Zambrana la referencia. 
se detiene en la importancia diegética que se le da a la capa sino que supone todo un valor dramático, tal y como el mismo narrador se encarga de precisarlo:

Sabido es que, así como en nuestros días ningún hombre que en algo se estima sale a la calle en mangas de camisa, así en los tiempos antiguos nadie que aspirase a ser tenido por decente osaba presentarse en la vía pública sin la respectiva capa. Hiciese frío o calor, el español antiguo y la capa andaban en consorcio, tanto en el paseo y el banquete cuanto en la fiesta de iglesia. ${ }^{6}$

La capa no era un simple aderezo sino que ella enunciaba, en el planteamiento palmino, un ascendiente social. Quien la poseía podía alternar en circunstancias tales como el paseo, el banquete y la misa (adviértase la cuidada manera que tiene el narrador de enunciar situaciones sociales diferentes y complementarias). En caso contrario, como ocurría con los caballeros de la capa, el individuo se exponía a la marginalización, o bien la "indecencia”. Por otro lado, se hace una alusión a lo que es espacio público (la "vía pública"), lo cual no debe ser obviado en la medida en que el primer lugar, el solar de San Millán, era más bien un espacio íntimo, privado. Entre el espacio privado, de intimidad miserable, y el espacio público, de exhibición social, se plantea una tensión violenta e irresuelta para los denominados caballeros de la capa. Dicha tensión es configurada, por el momento, de manera social pero no seguirá así por mucho tiempo pues casi de inmediato aparecerá el antagonista de los caballeros de la capa, el secretario de Francisco Pizarro, llamado Antonio Picado, "que ejerció sobre el marqués una influencia fatal y decisiva"7.

\footnotetext{
6 op.cit., p.138.

7 Ibíd., p.139.
} 
Antonio Picado es denominado por el narrador, generalmente circunspecto cuando se trata de sus personajes, como "demonio de perdición"8, lo que no deja de apuntalar la representación negativa del personaje. Será el precisamente quien se aprovechará de la protección de Francisco Pizarro para insultar públicamente a los almagristas: "llevando su provocación hasta el punto de que cuando algunos de ellos se asomaron, les hizo un corte de manga diciendo: -Para los de Chile -y picó espuelas al bruto"9. El gesto de Antonio Picado trasciende la intimidad del solar en el que se refugian los caballeros de la capa, elimina simbólicamente lo poco que conservaban de honor y les obliga a reaccionar de manera excesiva si es que quieren resarcir su imagen, ya de por sí precaria. De ahí que juren vengar la afrenta: "Juremos por la salvación de nuestras ánimas morir en la guarda de los derechos de Almagro el Mozo, y recortar de esta capa la mortaja para Antonio Picado"10. Si el insulto del secretario altera las relaciones entre el espacio de "adentro" y el de "afuera", el destino de la capa corre igual suerte al ser concebida como mortaja. El honor solamente se puede resarcir mediante el asesinato o, lo que es lo mismo, la eliminación física de aquello que lo ha mancillado.

\section{La casa del noble conquistador}

No se puede dejar de señalar la secreta simetría entre las partes que componen la Tradición. Así, si en la primera parte nos encontramos frente a un movimiento que desde adentro se abre hacia fuera, en la segunda es más bien lo inverso, es el afuera que termina invadiendo el espacio de adentro. Es en

8 Ibíd., p.138.

9 Ibíd., p.140.

10 Ibíd., p.141. 
esta dinámica que debemos entender el hecho de que Francisco Pizarro, en su calidad de autoridad, convoque a Juan de Rada, el tutor de Almagro el Mozo, al palacio. Quienes se reconocen como subordinados a un poder establecido, aunque sea por delegación, deben rendir cuentas en el espacio que lo encarna. De esta manera se abre la segunda parte con un gobernador que intenta quedar en buenos términos con quienes lo asesinarán, sin darse cuenta cabal de lo que se teje en contra suya:

Las cosas no podían concertarse tan en secreto que el marqués no advirtiese que los de Chile tenían frecuentes conciliábulos, que reinaba entre ellos una agitación sorda, que compraban armas y que, cuando Rada y Almagro el Mozo salían a la calle, eran seguidos, a distancia y a guisa de escolta, por un grupo de sus parciales. Sin embargo, el marqués no dictaba providencia alguna. En esta inacción del gobernador recibió cartas de varios corregimientos participándole que los de Chile preparaban sin embozo un alzamiento en todo el país. Esta y otras denuncias le obligaron una mañana a hacer llamar a Juan de Rada.

Encontró este a Pizarro en el jardín de palacio, al pie de una higuera que aún existe (...). ${ }^{11}$

Cuando se trataba de los caballeros de la capa, el grupo de hombres se guarecía en un solar; en cambio, el gobernador hace uso del palacio en el cual no se esconde sino que recibe en su calidad de funcionario real. Entre un espacio cualquiera, como el del solar, y el palacio se plantea una jerarquía consecuencia de las prebendas obtenidas por el conquistador, no necesariamente aceptadas acríticamente. Aquel hombre, otro hidalgo español de situación humilde, que se hizo de un destino en tierras americanas, es denostado y odiado por quienes vieron destruidas sus esperanzas de un futuro mejor.

11 Ibíd., p.142. 
Los almagristas, representados por Juan de Rada, ingresan a palacio como los derrotados que deben justificar sus acciones. Una nueva afrenta que se añade a la serie de malentendidos y discusiones entre un bando y otro.

Curiosamente, el Francisco Pizarro de la Tradición parece instalado en una situación en la que no advierte lo crítico del momento, razón por la cual deja de reaccionar con la rapidez y el rigor debidos: "el marqués no dictaba providencia alguna". Como si se tratase de una divinidad, Francisco Pizarro se encuentra en la capacidad de "dictar providencias", disposiciones que pongan término al conflicto político con los almagristas. Sin embargo, no manifiesta la clarividencia propia de las divinidades, sino más bien una ceguera muy humana y, si consideramos el momento, poco oportuna. Eso no impide que, por un efecto dramático, con el uso de la palabra "providencia" se enfatice en las alturas donde se encuentra el gobernador, en la medida en que páginas más adelante el mismo personaje será "echado abajo", derrocado por los rencores almagristas. Asimismo se anticipa, de un modo o de otro, el espacio de la entrevista entre él y Juan de Rada, el jardín del palacio.

Ambos no se encontrarán, como el protocolo lo hubiera esperado, en cualquier salón, sino en el jardín, una especie de epígono, continuando con la intertextualidad, tanto del Edén (Antiguo Testamento) como de Getsemaní (Nuevo Testamento). En el jardín, donde se encuentra la divinidad, curiosamente un espacio literariamente antes femenino que masculino ${ }^{12}$, se discutirá acerca de la sedición y las motivaciones almagristas. Dicha discusión sirve a Ricardo Palma para mostrar un Francisco

12 Para continuar con la intertextualidad bíblica, no podemos olvidar al Cantar de los cantares donde el jardín y el huerto presentados son feminizados en la amada: "Eres un jardín cerrado hermana mía, novia mía; eres un jardín cerrado, una fuente sellada" (4:12). 
Pizarro comprensivo, benevolente e incluso generoso: “le obsequió Pizarro seis higos que él mismo cortó por su mano del árbol"13. La alusión al jardín, pese a su brevedad, carga semánticamente el espacio confiriéndole no solo un aliento bíblico sino también político. Los higos no dejan de recordar al árbol del jardín del Edén, árbol del conocimiento y de la sabiduría. Lamentablemente, para Francisco Pizarro no existe conocimiento alguno sino todo lo contrario, una profunda ignorancia de lo que se está tramando contra él, lo cual supone una ironía por la parte del autor. Por otro lado, no se trata de un manzano sino de un higuero, un árbol que en la tradición occidental simboliza la ciencia y la fertilidad ${ }^{14}$.Si bien el primer valor concuerda con el guiño al Jardín del Edén, el segundo apunta en el sentido irónico de la situación pues, sin querer agotar las interpretaciones, podemos decir que con sus higos Francisco Pizarro le entrega la "vida" a quien le devolverá la muerte. Decimos, por lo demás, aliento político pues el virrey entrega a Juan de Rada seis higos, el mismo número de sillones que hay en el solar. Los higos no alcanzarán para todos los descontentos hidalgos cuyo número es el doble. Se trata de un regalo inconsciente de Francisco Pizarro que desnuda el porqué de las tensiones: un considerado mal reparto del botín y las prebendas.

A Francisco Pizarro no le sirve de nada el encuentro con el mentor de Almagro el Mozo. Inmediatamente después el narrador se detiene en el asesinato del marqués. La representación de Francisco Pizarro pareciera colocarlo moralmente por encima de quienes lo asesinaron. No solo luchó en desventaja contra

13 Op.cit., p.143.

14 Así se explica en el Dictionnaire des symboles dirigido por Jean Chevalier: "Avec l'olivier, la vigne, le figuier est un des arbres qui symbolisent l'abondance”. Poco más adelante se añade que: "Le figuier symbolise la science réligieuse". En: CHEVALIER Jean (Dir.), Dictionnaire des symboles, Paris, Robert Laffont, 1969, p.352. 
ellos -lo cual no puede más que enaltecerlo- sino que también se muestra como un buen cristiano pues al final llega a hacer una cruz con su sangre en la tierra: "El conquistador del Perú solo pronunció una palabra: iJesús!, y cayó, haciendo con el dedo una cruz de sangre en el suelo, y besándola"15. Podemos decir que el narrador de Los caballeros de la capa, a diferencia del metomentodo que Palma utiliza en otras tradiciones, se mantiene relativamente discreto en esta, pero lo que no se puede negar es el parti pris del autor por Francisco Pizarro, quien hasta en el momento de su muerte se encargará de subrayar su coraje, entrega y devoción. En el universo colonial representado, el marqués Pizarro es la imagen de un gobernador ideal, asesinado por fuerzas irracionales y abyectas: "Quisieron más tarde sacar el cuerpo de Pizarro y arrastrarlo por la plaza; pero los ruegos del obispo de Quito y el prestigio de Juan de Rada estorbaron este acto de bárbara ferocidad"16. Así, termina la vida de un hombre que, tal y como lo presenta el autor, pudo haber sembrado la semilla de la civilización y piedad en tierras americanas.

Finalmente, continuando con la simetría entre las partes, si la primera sección se terminaba con el juramento hecho por los hidalgos de vengarse de la afrenta efectuada por Picado, la segunda termina con la resolución de este juramento en un espacio público. A la afrenta social le sigue su venganza en la plaza, lo cual podría resolver el conflicto del argumento. No obstante, la tercera parte muestra la restitución del orden social y político mediante la representación de un nuevo y último espacio en el cual ya no se enfrentarán dos bandos sino que, en una precipitación de la violencia, se chocarán los almagristas contra las fuerzas reales.

15 op.cit., p.145.

16 op.cit., p.145. 


\section{El espacio abierto de la resolución social}

Apenas empieza la tercera y última parte, lo primero que llama la atención son las palabras del narrador:

No nos proponemos entrar en detalles sobre los catorce meses y medio que Almagro el Mozo se mantuvo como caudillo, ni historiar la campaña que, para vencerlo, tuvo que emprender Vaca de Castro. Por eso, a grandes rasgos hablaremos de los sucesos. Con escasas simpatías entre los vecinos de Lima, viose don Diego forzado a abandonar la ciudad para reforzarse en Guamanga y el Cuzco, donde contaba con muchos partidarios. Días antes de emprender la retirada, se le presentó Francisco de Chaves exponiéndole una queja, y no recibiendo reparación de ella le dijo: «No quiero ser más tiempo vuestro amigo, y os devuelvo la espada y el caballo». Juan de Rada lo arrestó por la insubordinación, y enseguida lo hizo degollar. Así concluyó uno de los caballeros de la capa. ${ }^{17}$

Contra todo pronóstico, el narrador anuncia que no continuará con esa manera minuciosa de contar las tensiones entre bandos rivales: de ahí se explica que las dos primeras partes desarrollen eventos ocurridos en apretados tres meses (del 5 de junio al 29 de septiembre de 1541), mientras que la tercera se extiende a lo largo de tres años; es decir, de septiembre de 1541 a 1544, la fecha en que muere el último de los caballeros de la capa. Lo importante no es el destino de aquellos que con su conducta desafiaron al poder colonial, destinos evacuados rápidamente; antes bien, lo que cuenta de verdad es la restitución del orden por parte de las autoridades reales. En otros términos, la tercera parte se detendrá en la manera en que la violencia oficial y legal se impone para restituir el correcto curso de los

17 op.cit., p.148. 
acontecimientos tanto a nivel político como administrativo: "Estaba escrito que todos habían de morir de muerte violenta y bañados en su sangre"18. No deja de resultar curioso que con la alusión a la escritura se subraye el carácter ineluctable de los acontecimientos y también la ejecución de la legalidad, esa palabra escrita que prescribe las conductas.

Por eso, el narrador precisa desde el inicio que Almagro el Mozo abandonó Lima. El espacio en el cual se desarrolló la historia en las dos primeras partes termina siendo cambiado por otros lugares "periféricos" en términos administrativos; nos referimos a las provincias de Huamanga y Cuzco. Ricardo Palma configura el espacio de la Ciudad de los Reyes como favorable al gobernador asesinado y enemigo de los de la capa. En el texto, los vecinos limeños son homogéneamente ciudadanos fieles del orden, sujetos celosos por deslindar responsabilidades (lo cual sigue enfatizando la nula corrección de los asesinos). Eso explica el hecho que Almagro el Mozo se acerque a espacios hostiles al orden colonial como Huamanga y Cuzco, lugares en los cuales se dieron numerosas sublevaciones indígenas. De ahí que no sea casual que, hacia el final del relato, Diego Méndez y Gómez Pérez se asilen en Vilcabamba, Cuzco, donde se refugia Manco Inca, otro rebelde del poder establecido. Los españoles que asesinaron a Francisco Pizarro no dudan ningún momento en aliarse con los enemigos declarados del poder colonial. En la representación de Ricardo Palma los indígenas, al ser colocados del lado de los asesinos, resultan ser cómplices de los delincuentes. No podemos dejar de reconocer, por lo tanto, la manera sutil con que el escritor limeño se alinea en los rangos hispanizantes, restándole a los incas de Vilcabamba cualquier legitimidad política.

18 op.cit., p.148. 
Es necesario añadir que en la actitud de Francisco de Chaves se formula y anuncia lo que será la conducta de los otrora enemigos de Francisco Pizarro. Una vez desaparecido el virrey y asesinado Picado, ya nada los reuniría frente en un territorio acéfalo de poder. La discordia y la inquina aparecen entre ellos para hacer que se odien y se traicionen mutuamente. Si en el palacio de Pizarro, Juan de Rada había aparecido como Judas delante de Jesús en Getsemaní, lo cual anunciaba la temática de la traición, ahora se lleva al extremo este rasgo pues los doce caballeros llevan la felonía en la sangre. Incapaces de mantenerse unidos, sucumben lentamente a los esfuerzos de Vaca de Castro por restablecer el orden colonial mientras "se aproximaba el momento decisivo"19.

El momento decisivo no es otro que el del domingo 16 de septiembre de 1542, cuando tuvo lugar la batalla de Chupas. Pese a que no existe descripción alguna del campo de batalla, el lector reconocerá en la evacuación total de espacios ya conocidos en el texto, como los solares (primera parte) o los palacios (segunda parte), el deseo de reducir la resolución del conflicto a un espacio bélico y abierto. Ya no existen los lugares de las intrigas, los honores heridos y las infidencias a media voz; en lugar de ellos encontramos un espacio en el que los héroes y los mártires, junto con los traidores, luchan a muerte por el deseo regio de restablecer el óptimo funcionamiento con respecto de los intereses monárquicos. Eso explica, en última instancia, que Vaca de Castro no se haya contentado con ganar sobre el terreno de batalla sino que se también haya buscado el "espectáculo" del poder restablecido mediante ajusticiamientos que no dejan de tener un sentido poético, a juzgar por las palabras de Almagro el Joven: "muero en el lugar donde degollaron a mi padre, ruego solo que me coloquen en la misma

19 op.cit., p.149. 
sepultura debajo de su cadáver" ${ }^{20}$.Donde se enterró al padre se enterró al hijo, concluyendo de esa manera la sedición de los caballeros de la capa y el relato de Ricardo Palma, como si, pese a todo, la política y la familia se explicaran y reclamaran mutuamente.

\section{Conclusiones}

Ricardo Palma se sirve de hechos históricos para interrogar el momento, frágil, inestable, en el que se estaba instalando un poder colonial en las tierras americanas. No lo hace como historiador sino como literato $y$, en ese sentido, insufla una carga simbólica a los espacios en los cuales se desarrollan sus tradiciones. En el caso específico de Los caballeros de la capa, develamos las simetrías narrativas que juegan en paralelo con la constitución y la interacción de los espacios. Las tensiones entre los espacios cerrados y los espacios abiertos, los espacios privados y los espacios públicos, son declinadas a diversos grados y registros con el objetivo de darle una densidad narrativa al relato. Al mismo tiempo, la literatura permite a Ricardo Palma un posicionamiento moral y político singular. Pese a haber abandonado la política activa en $1872^{21}$, Ricardo Palma nunca dejó de lado sus inquietudes sociales y políticas, haciendo de la literatura un terreno de memoria y combate. Por eso, el pasado le interesa pero no como si se tratase de un objeto de colección, totalmente desconectado del presente, sino por todo lo que de

20 op.cit., p.152.

21 Así lo formula el crítico José Miguel Oviedo: "Pero Palma ya no es el mismo: los sangrientos hechos, la muerte brutal de su amigo Balta, la violencia destructora de la lucha por el poder, han dejado en él huellas imborrables. Él que había firmado aquella tarde del 22 de julio una propuesta del senado contra el golpe de los Gutiérrez, ha perdido toda fe e interés en la política”. En: OVIEDO José Miguel, Genio y figura de Ricardo Palma, Buenos Aires: Editorial Universitaria, 1965, p.82. 
contemporáneo existe en él. Esto explica la frase con la que abrimos este artículo, y que nos sirvió de epígrafe, acaso la que mejor encarna la perspectiva de Ricardo Palma: "Caídos y levantados, hartos y hambrientos, eso ha sido la colonia y eso es y ha sido la república". Desencantado de la actividad política, aunque comprometido con el derrotero nacional el autor de las Tradiciones peruanas se vale de la literatura para recrear un pasado $\mathrm{y}$, mediante la literatura, entregarle un significado contemporáneo de claro sello hispanizante, de castizo motivo e intención.

\section{Bibliografía}

ANÓNIMO, Lazarillo de Tormes, Madrid : Cátedra, 1192, 336 p.

BARTHES Roland, L'effet de réel, Communications. 1968, vol 11 , n'll, p.84-89.

CHEVALIER Jean (Dir.), Dictionnaire des symboles, Paris, Robert Laffont, 1969, 844 p.

OVIEDO José Miguel, Genio y figura de Ricardo Palma, Buenos Aires: Editorial Universitaria, 1965, 192 p.

PALMA Ricardo, Tradiciones peruanas (selección), Madrid: Cátedra, 2011, 635 p.

QUEVEDO Francisco de, La vida del Buscón, Barcelona: Crítica, 1993, 437 p.

TAUZIN-CASTELLANOS Isabelle, Las Tradiciones Peruanas de Ricardo Palma: claves de una coherencia, Lima: Universidad Ricardo Palma, 1999, 234 p. 